\title{
An Energy-Efficient Access Control Algorithm with Cross-Layer Optimization in Wireless Sensor Networks
}

\author{
Zhi Chen, Shaoqian Li \\ National Key Laboratory of Communication, University of Electronic Science and Technology, Chengdu, China \\ E-mail: chenzhi@uestc.edu.cn \\ Received July 20, 2009; revised October 25, 2009; accepted November 17, 2009
}

\begin{abstract}
This paper presents a wireless sensor network (WSN) access control algorithm designed to minimize WSN node energy consumption. Based on slotted ALOHA protocol, this algorithm incorporates the power control of physical layer, the transmitting probability of medium access control (MAC) layer, and the automatic repeat request (ARQ) of link layer. In this algorithm, a cross-layer optimization is preformed to minimizing the energy consuming per bit. Through theory deducing, the transmitting probability and transmitting power level is determined, and the relationship between energy consuming per bit and throughput per node is provided. Analytical results show that the cross-layer algorithm results in a significant energy savings relative to layered design subject to the same throughput per node, and the energy saving is extraordinary in the low throughput region.
\end{abstract}

Keywords: Wireless Sensor Network (WSN), Cross-Layer Design, Energy Efficient, Energy Consumption per Bit, Throughput

\section{Introduction}

Wireless sensor network (WSN) consist of a large number of small, low data rate and inexpensive node that communicate in order to sense or control a physical phenomenon. Most of the applications proposed for WSN depend on node designs that minimize complexity and energy consumption [1]. This is because WSN node battery replacement will be difficult due to deployment in remote locations or in difficult environment. Even when nodes are accessible, their low cost may make it more efficient to simply replace the entire node rather than just its battery.

Opportunities for minimizing WSN node energy consumption exist at all layers of the protocol state. Many of the proposed WSN routing algorithm account for energy consumption in some fashion [2]. Considerable work has also been performed on minimizing energy consumption at the medium access control (MAC) layer [3,4]. Energy can also be saved at the physical layer by optimizing parameters such as modulation scheme and number of transmit antennas $[5,6]$.

While working with individual protocol layers will

This work is supported in part by Key Projects in National Science \& Technology Program under Grant 2008ZX03005-001 help to conserve WSN energy, it has been shown that cross-layer optimization of the entire protocol stack will result in the greatest savings [7]. However, computing this optimal design and implementing it across a WSN is difficult due to the strict complexity constraints imposed on the WSN nodes. As a result, researchers have started to investigate simplified cross-layer energy conservation solutions that are more suited to low complexity WSN devices [8-10].

In $[8,9]$, promising results have been presented for minimizing WSN energy consumption using across-layer power control algorithm that accounts for link layer and physical layer behavior. This approach balances the energy consumed by the physical layer hardware with the energy consumed by frames retransmitted as part of an automatic repeat request (ARQ) error recovery scheme. The basic idea is that lowering transmit power decreases physical layer energy consumption but a transmit power that is too low increases the energy wasted on excess ARQ retransmissions. As a result, an optimal transmit power exists that achieves a compromise between these two effects. One drawback to the work presented in $[8,9]$ is that the physical layer model used in both papers assumes only thermal noise in the radio channel and does not include multiple access interference (MAI). When the SNR is high, the MAI effect on the BER can't be 
ignored, so the optimized transmit power level presented in $[8,9]$ would not be the actual optimized value.

Based on the work of $[8,9]$, a new power control algorithm that accounts for MAI and MAC layer behavior is proposed in [10]. The contribution of [10] is that the MAI effect on physical BER is taken into account, and an accurate queuing system is used to model for ALOHA MAI. Although how physical layer reliability is affected by the MAI is considered, the parameter designs of MAC layer, such as transmit probability, are not involved in the cross-layer optimization in [10]. Meanwhile, the comparisons of energy consumption alone are not sufficient, because the energy consumption per bit is always relative to the throughput per node. So the trade-off between the energy consumption per bit and the throughput per node should be taken into consider.

The contribution of this paper is to present a new cross-layer access control (CLAC) algorithm that accounts for power control of physical layer, access control of MAC layer, and ARQ behavior of link layer. Using the CLAC algorithm, a significant energy savings relative to layered algorithms can be achieved subject to the same throughput demand.

\section{The System Model}

A star network topology is assumed where each sensor node transmits its send information to a sink node. Although the star network showed in Figure 1 is very simple, it could represent a complicate mesh network where the routing algorithm forms clusters with all nodes in cluster transmitting to a central node designated as the cluster head.

In the link layer, the ARQ mechanism is adopted. For the simplification of analysis, the overhead of packet header and CRC in front channel, the error and delay in back channel are all ignored.

In the MAC layer, slotted ALOHA is selected as the

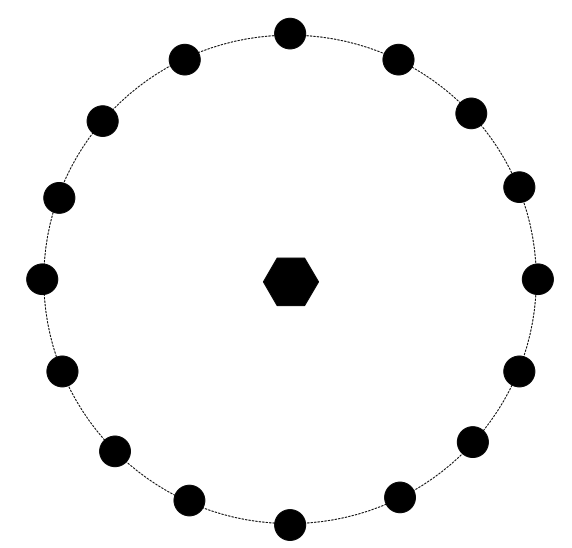

Figure 1. A star topology for WSN. random access protocol. Each sensor node has the nodes transmit signals in the same frequency band, and the band width is $B$. Each node transmits information at a fixed rate of $R$. The signal transmit power and the noise power are denoted by $P$ and $P_{N}$ respectively, and hence the transmit $\mathrm{SNR}=P / P_{N}$. We assume a Rayleigh block fading channel for every sensor node in every slot. The fading gain for user $i$ in the time slot $t$ is denoted by $h_{i}(t)$ and is assumed to have unit variance. The fading is also assumed to be independent across users and slots, so $h_{i}$ can be used to define the fading gain for sensor node $i$ in any time slot. The sink node employs single user decoding to decode information from sensor nodes. By that we mean the sink node decode one sensor's codeword assuming ever other sensor node's signal as noise. In order not to be constrained with a special modulate scheme, outage probability $\varepsilon$ is used to describe the transmitting error. The outage probability of $k$ sensor nodes transmit in a slot is [12].

$$
\varepsilon=1-\operatorname{Pr}\left\{R \leq B \log \left(1+\frac{\left|h_{1}\right|^{2} \mathrm{SNR}}{\sum_{i=2}^{k}\left|h_{i}\right|^{2} \mathrm{SNR}+1}\right)\right\}
$$

When the fading coefficients are i.i.d. Rayleigh with unit variance, then

$$
\varepsilon=1-\exp \left(-\frac{\left(2^{R / B}-1\right)}{\mathrm{SNR}}\right) 2^{-R(k-1) / B}
$$

From (2), it is obviously that the outage probability $\varepsilon$ is relative to SNR and $k$. In this scheme, the throughput is given by

$$
\rho(\mathrm{SNR}, k)=(1-\varepsilon) R=R \times \exp \left(-\frac{\left(2^{R / B}-1\right)}{\mathrm{SNR}}\right) 2^{-(k-1) R / B}
$$

The sensor node has small bulk and simple functions, most of the energy is consumed by the signal transmittting, so the circuit energy consumption is ignored in our analysis. The energy consumption per bit involves the energy consumed by the physical layer signal transmitting and the energy consumed by link layer frames retransmitting. The energy consumption per bit with the scheme of SNR and $k$ is given by

$$
E(\mathrm{SNR}, k)=P /[R(1-\varepsilon)]=(P / R) \times \exp \left(\frac{2^{R / B}-1}{\mathrm{SNR}}\right) 2^{(k-1) R / B}
$$

The number $k$ of sensor nodes transmitting in a slot 
is relate to the transmits probability $p$, the throughput and energy consumption per bit can be computed by averaging $\rho(\mathrm{SNR}, k)$ in (3) and $E(\mathrm{SNR}, k)$ in (4) by $k$ [12]. The results are given as follows

$$
\begin{aligned}
& \rho(\mathrm{SNR}, p)=\sum_{k=1}^{N}\left(\begin{array}{l}
N \\
k
\end{array}\right) p^{k}(1-p)^{N-k} \rho(\mathrm{SNR}, k) \\
= & \left(2^{-R / B} p+1-p\right)^{N-1} p N R \exp \left(-\frac{2^{R / B}-1}{\mathrm{SNR}}\right) \\
E & (\mathrm{SNR}, p)=\sum_{k=1}^{N}\left(\begin{array}{l}
N \\
k
\end{array}\right) p^{k}(1-p)^{N-k} E(\mathrm{SNR}, k) \\
= & \left(2^{R / B} p+1-p\right)^{N} P / R \exp \left(\frac{2^{R / B}-1}{\mathrm{SNR}}\right) 2^{-R / B}
\end{aligned}
$$

\section{Cross-Layer Access Control Algorithm}

According to (5) and (6), the optimization problem of minimizing energy consumption per bit can be described as follow, subject to the throughput demand $\rho$, the optimal transmit power level $P$ and transmit probability $p$ should be decided to achieve the minimal energy consumption per bit $E$. This is a nonlinear programming problem with constraints, the straightforward solution is much complicated, which is not suited to low complexity WSN devices. Therefore, a simplified optimization algorithm should be developed for sensor nodes.

The partial derivative of function $E(\mathrm{SNR}, p)$ with respect to argument $p$ is computed as

$$
\begin{aligned}
& \frac{\partial E(\mathrm{SNR}, p)}{\partial p}=\left(2^{R / B} p+1-p\right)^{N} P / R \exp \left(\frac{2^{R / B}-1}{\mathrm{SNR}}\right) 2^{-R / B} \\
& =N\left(2^{R / B} p+1-p\right)^{N-1}\left(2^{R / B}-1\right) P / R \exp \left(\frac{2^{R / B}-1}{\mathrm{SNR}}\right) 2^{-R / B}>
\end{aligned}
$$

According to (7), $E(\mathrm{SNR}, p)$ is a monotonic increasing function of $p$. Similarly, the partial derivative of function $E(\mathrm{SNR}, p)$ with respect to argument $P$ is computed as

$$
\begin{aligned}
& \frac{\partial E(\mathrm{SNR}, p)}{\partial P}=\left(2^{R / B} p+1-p\right)^{N} P / R \exp \left(\frac{\left(2^{R / B}-1\right) P_{N}}{P}\right) 2^{-R / B} \\
& =\frac{\left(2^{R / B} p+1-p\right)^{N} 2^{-R / B} \exp \left(\frac{2^{R / B}-1}{P / P_{N}}\right)}{R}\left(1-\frac{\left(2^{R / B}-1\right) P_{N}}{P}\right)
\end{aligned}
$$

According to (8), there exists an optimal solution of $P$ to achieve the minimal $E(\mathrm{SNR}, p)$. By solving the Equation $\frac{\partial E(\mathrm{SNR}, p)}{\partial P}=0$, the optimal solution of $P$ is given by

$$
\tilde{P}=\left(2^{R / B}-1\right) P_{N} \quad, \quad \text { or } \mathrm{SNR}=2^{R / B}-1
$$

It is obvious that $\tilde{P}$ is independent of the value of $P$.

In the same way, by solving the partial derivative of function $\rho(\mathrm{SNR}, p)$ with respect to arguments $P$ and $P$, it is found that $\rho(\mathrm{SNR}, p)$ is a monotonic increasing function of $P$, and there exists an optimal solution of $P$ to achieve the maximal $\rho(\mathrm{SNR}, p)$. The optimal solution of $P$ is given by

$$
\tilde{p}=\frac{1}{N\left(1-2^{-R / B}\right)}
$$

Note that $\tilde{p}$ is independent of the value of $P$.

Substituting $P$ and $p$ using relevant optimal value $\tilde{P}$ and $\tilde{p}$ in (5) and (6), we get the cross-layer optimized throughput and energy consumption per bit as follows

$$
\begin{aligned}
& \tilde{\rho}=\frac{R}{1-2^{-R / B}} e^{-1}\left(1-\frac{1}{N}\right)^{N-1} \\
& \tilde{E}=\frac{2^{R / B(N-1)}\left(2^{R / B}-1\right) P_{N} e}{N^{N} R}
\end{aligned}
$$

The low energy consumption performance of this CLAC algorithm is analyzed by comparing with the traditional layered access control (TLAC) algorithm in which the transmit power and the transmit probability is determinate independently.

In TLAC algorithm, the power control balances the energy consumed by the physical layer and the link layer, therefore, the Equation $\mathrm{SNR}=2^{R / B}-1$ is satisfied still. The difference of TLAC from CLAC is that the transmit probability $P$ is independent of the value of SNR. The throughput and energy consumption per bit in TLAC algorithm are given by

$$
\begin{gathered}
\rho^{\prime}=\left(2^{-R / B} p+1-p\right)^{N-1} p N R e^{-1} \\
E^{\prime}=\frac{\left(2^{R / B} p+1-p\right)^{N} e\left(2^{R / B}-1\right) P_{N} 2^{-R / B}}{R}
\end{gathered}
$$

\section{Comparison of Layered and Cross-Layer Approaches}

Because there exists the trade-off between the throughput 
and energy consumption per bit, the plot of energy consumption per bit versus the throughput can compare the energy saving performance of CLAC and TLAC fairly and clearly.

In order to generate this plot, some assumptions must be made about the parameters of the WSN. It is assumed that the WSN consist of $N=16$ sensor nodes. A data rate of $R=20 \mathrm{kbps}$, a noise power of $P_{N}=-110 \mathrm{dbmw}$ and a frequency bandwidth of $B=20 \mathrm{kHz}$ are chosen for the physical layer. A transmit probability $p=1 / 16$ is used for TLAC. The resulting energy consumption per bit versus the throughput plot is shown in Figure 2. The upper curve denotes the TLAC algorithm and the lower curve denotes the CLAC algorithm.

Figure 2 shows that CLAC consumes less energy per bit than TLAC at the same throughput demand. In the high throughput region, two curves are close to each other. The SNR increase along with the increase of throughput, the MAI has more evident influence on BER than noise. This evident influence of MAI result in that the transmit probability designs can ignore the value of SNR, so the performances of two algorithm are close. On the other hand, CLAC have significant energy savings relative to TLAC in the low throughput region. The SNR decrease along with the decrease of throughput, the effect of noise on BER is obvious, so it is very necessary for the transmit probability variety according to SNR.

Another interesting phenomenon can be found in Figure 2. For CLAC, the energy consumption per bit decreease as the decreasing of throughput, but the energy consumption per bit for TLAC trended to be constant as the decreeasing of throughput. The reason is that the transmit probability for TLAC dose not change along with SNR, the multiple access channel capacity is not used adequately, and the decreasing of SNR and the decreasing of data rate have canceling effect on the energy consumption per bit.

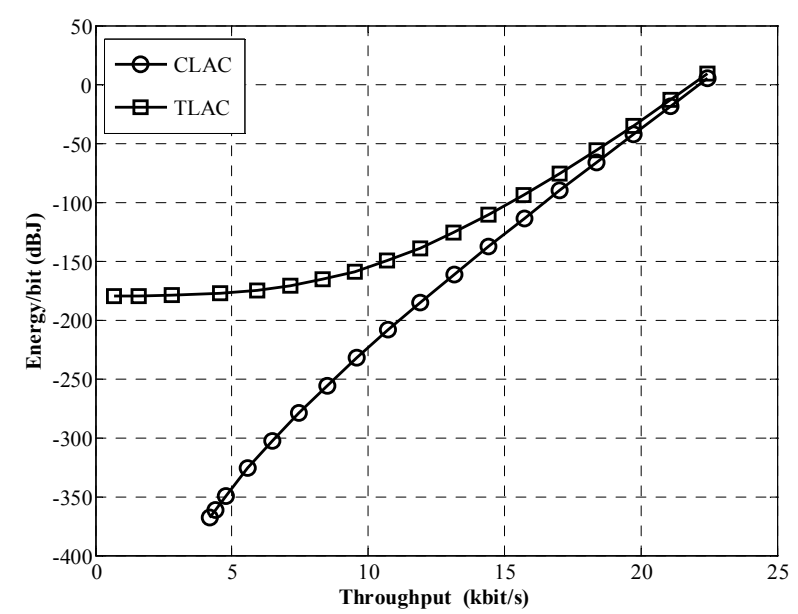

Figure 2. Energy consumption per symbol vs. throughput per node.
It also emphasized that the cross-layer optimizes access control is indispensable for low throughput WSN.

\section{Conclusions}

This paper presents a WSN access control algorithm. Based on slotted ALOHA protocol, this algorithm incorporates the power control of physical layer, the transmitting probability of MAC layer, and the ARQ of link layer. In this algorithm, a cross-layer optimization is preformed to minimizing the energy consuming per bit. Analytical results show that the cross-layer algorithm results in a significant energy savings relative to layered design subject to the same throughput per node. At the same time, the cross-layer algorithm has low complexity of implementation, and it is suitable to the low energy consumption and low complexity WSN devices.

\section{References}

[1] K. Romer and F. Mattern, "The design space of wireless sensor networks," IEEE Wireless Communications, Vol. 11, No. 6, pp. 54-61, 2004.

[2] J. N. Al-Karaki, and A. E. Kamal, "Routing techniques in wireless sensor networks: A survey," IEEE Wireless Communications, Vol. 11, No. 6, pp. 6-28, 2004.

[3] Y. E. Sagduyu and A. Ephremides, "The problem of medium access control in wireless sensor networks," IEEE Wireless Communications, Vol. 11, No. 6, pp. 4453, 2004.

[4] W. Ye, J. Heidemann, and D. Estrin, "Medium access control with coordinated adaptive sleeping for wireless sensor networks," IEEE/ACM Transactions Networking, Vol. 12, No. 3, pp. 493-506, 2004.

[5] S. Cui, A. J. Goldsmith, and A. Bahai, "Energy-constrained modulation optimization," IEEE Transactions Wireless Communications, Vol. 4, No. 5, pp. 2349-2360, 2005.

[6] S. Cui, A. J. Goldsmith, and A. Bahai, "Energy-efficiency of MIMO and cooperative MIMO techniques in sensor networks," IEEE Journal on Selected Areas in Communications, Vol. 22, No. 6, pp. 1089-1098, 2004.

[7] J. Goldsmith and S. B. Wicker, "Design challenges for energy constrained ad hoc wireless networks," IEEE Wireless Communications, Vol. 9, No. 4, pp. 8-27, 2002.

[8] M. De Sanctis, E. Cianca, and M. Ruggieri, "Energy efficient transmit power control for HDR WPAN," in Proceeding'06 17th IEEE International Symposium on Personal, Indoor and Mobile Radio Communications, PIMRC'06, pp. 1-5, 2006.

[9] Howitt and J. Wang, "Energy efficient power control policies for the low rate WPAN," in Proceeding'04 IEEE Conference on Sensor and Ad Hoc Communications and Networks, SECON'04, pp. 527-536, 2004.

[10] G. M. Geoffrey, A. H. Jennifer, and J. D. Robert, “A sensor network cross-layer power control algorithm that 
incorporates multiple-access interference," IEEE Transactions on Wireless Communications, Vol. 7, No. 8, pp. 2877-2883, 2008.

[11] L. Tong, Q. Zhao, and G. Mergen, "Multi packet reception in random access wireless networks: from signal processing to optimal medium access control $[\mathrm{J}]$,"
IEEE Communications Magazine, Vol. 39, No. 11, pp. 108-12, 2001.

[12] V. Naware and L. Tong, "Cross layer design for multiaccess communication over rayleigh fading channels," IEEE Transactions on Wireless Communications, Vol. 7, No. 3, pp. 1095-103, 2008. 